\title{
INTERFERENCIA DE LA VACUNACIÓN SIMULTÁNEA CONTRA METAPNEUMOVIRUS AVIAR, BRONQUITIS INFECCIOSA Y ENFERMEDAD DE NEWCASTLE EN POLLOS DE CARNE
}

\section{Interference of Simultaneous Vaccination Against Avian Metapneumovirus, InfeCtious Bronchitis, ANd Newcastle Disease in B ROILERS}

\author{
Víctor Palomino T. ${ }^{1}$, E. Icochea D. ${ }^{1,2}$, John Guzmán G. ${ }^{1}$, Rosa Sam T. ${ }^{3}$, \\ Alberto Manchego S. ${ }^{3}$
}

\section{RESUMEN}

\begin{abstract}
El estudio evaluó la interferencia en la protección contra la enfermedad de Newcastle (ENC) ocasionada por la vacunación contra Metapneumovirus (aMPV) en un programa de vacunación simultánea al primer día de edad contra ENC y Bonquitis Infecciosa (BI) en pollos de carne. Se usaron 400 pollos de carne Cobb-Vantress 500 divididos en cuatro grupos. El grupo I vacunado al primer día contra BI (H120), ENC (VG/GA) y aMPV (11/94) y revacunado al día 9 contra ENC (VG/GA). El Grupo II vacunado al primer día contra BI (Ma5), ENC (C2) y aMPV (11/94) y, revacunado al día 9 contra ENC (Clone 30). El grupo III vacunado al primer día contra BI (H120) y ENC (VG/GA) y revacunado al día 9 contra ENC (VG/GA). El Grupo IV no fue vacunado. Todos los grupos fueron desafiados el día 30 con una cepa velogénica viscerotrópica del virus de ENC. Se valuó mortalidad, signos clínicos, respuesta serológica, reacción post vacunal y parámetros productivos. La mortalidad fue de 12 y $16 \%$ para los grupos vacunados contra aMPV versus $23 \%$ para el grupo no vacunado contra aMPV, y $88 \%$ para el control sin vacunas; sin embargo, las diferencias entre los tres grupos vacunados no fueron significativas. Los resultados indican que la vacunación contra aMPV en pollos de carne no ocasionó interferencia en la protección contra la ENC en ninguno de los grupos vacunados simultáneamente contra la ENC, BI y aMPV al primer día de edad, y se vio menor reacción post vacunal usando la cepa entérica que con la cepa de tropismo respiratorio del virus de la ENC.
\end{abstract}

Palabras clave: Metapneumovirus Aviar, Bronquitis Infecciosa, Enfermedad de Newcastle, vacunación, desafío

\section{Abstract}

The study evaluated the interference in the immune protection system against Newcastle disease (ENC) caused by the vaccination against Avian Metapneumovirus (aMPV) in a simultaneous vaccination against ENC and Infectious Bronchitis (BI) in oneday-old broilers. Four hundred Cobb-Vantress 500 broilers were distributed into four

\footnotetext{
${ }^{1}$ Laboratorio de Patología Aviar, ${ }^{3}$ Laboratorio de Microbiología y Parasitología Veterinaria, Facultad de Medicina Veterinaria, Universidad Nacional Mayor de San Marcos, Lima

${ }^{2}$ E-mail : eliana.icochea@gmail.com
} 
groups. Group I vaccinated at 1-day-old against BI (H120), ENC (VG/GA) and aMPV (11/ 94) with revaccination at day 9 against ENC (VG/GA). Group II vaccinated at 1-day-old against BI (Ma5), ENC (C2) and aMPV (11/94) and revaccinated at day 9 against ENC (Clone 30). Group III vaccinated at 1-day-old against BI and ENC (VG/GA) and revaccinated at day 9 against ENC (VG/GA). Group IV received no vaccine. All groups were challenged at day 30 with a velogenic viscerotropic strain of ENC virus. Mortality, clinical signs, serologic response, post-vaccination reaction, and productive performance were evaluated. Mortality was 12 and $16 \%$ for groups vaccinated against aMPV versus $23 \%$ for the non-vaccinated group against aMPV, and $88 \%$ for the control group; however, no significant difference was found between the three vaccinated groups. Findings indicate that vaccination against aMPV in broilers did not cause interference in the protection against ENC in any of the vaccinated groups; besides, there was less post-vaccination reaction with the enteric strain vaccine instead of a respiratory strain of ENC.

Key words: Avian Metapneumovirus, Infectious Bronchitis, Newcastle Disease, vaccination, challenge

\section{INTRODUCCIÓN}

El Síndrome de Cabeza Hinchada $(\mathrm{SCH})$ en pavos y pollos de carne es una enfermedad de etiología multifactorial, en la que intervienen Metapneumovirus aviar (aMPV) y Escherichia coli. Reviste gran importancia económica ya que limitan la capacidad del ave para convertir alimento en carne. Fue observada por primera vez en Sudáfrica en 1979 (Morley y Thompson, 1984). De allí se extendió a Europa y América del Sur, y en 1997 a USA. En el Perú, la observación en campo de cuadros respiratorios acompañados por celulitis facial coincidió con la importación de pollos beneficiados procedentes de Francia en 1985. Además, ha sido observado en lotes de aves reproductoras y ponedoras comerciales (Icochea, 2002).

La exposición al aMPV causa una infección aguda del tracto respiratorio alto, caracterizada por tos, descarga nasal y conjuntivitis; además, en pollos de carne se produce edema subcutáneo en la cabeza, cara y debajo de la mandíbula, ocasionando una hinchazón de la cabeza con inflamación de parpados y oclusión de ojos. Esta condición se asocia frecuentemente a una infección se- cundaria por $E$. coli, que es responsable del material caseoso encontrado en el subcutáneo, oído medio y huesos craneanos, dando lugar a una tortícolis. La morbilidad es cercana al $10 \%$ y la mortalidad usualmente no sobrepasa el 2\% (Jones, 2002), aunque en casos complicados con E. coli puede llegar al 20\% (Gross, 1994). No está demostrada la transmisión vertical (Bennett et al., 2001), pero se ha detectado ARN viral en la fisura palatina de pavitos recién nacidos (Shin et al., 2002); asimismo, las progenies provenientes de reproductoras con brotes de aMPV tienen mayor tendencia a la enfermedad que progenies de reproductoras sanas (Villegas, 2009).

La prevención y control de la enfermedad están orientados a evitar la diseminación del virus y el estrés de las aves, mejorando la ventilación, reduciendo la densidad poblacional, evitando fluctuaciones de temperatura, implementando medidas de bioseguridad y utilizando el sistema de crianza "Todo dentro, todo fuera". Por tratarse de una enfermedad viral, el tratamiento es para evitar las infecciones secundarias ocasionadas principalmente por E. coli. Debido a la importancia económica de la enfermedad, se tiene que recurrir a la vacunación, aunque se han descrito interferencias entre virus 
vacunales respiratorios contra Bronquitis Infecciosa (BI) y la enfermedad de Newcastle (ENC) con la de aMPV (Cook, 2001; Ganapathy et al., 2006), de allí que algunos autores recomiendan abstenerse de vacunar pollos de carne contra aMPV (Jones, 2004).

En el 2006 entraron al mercado nuevas vacunas vivas contra aMPV diseñadas para pollos de carne que no producirían interferencia con otros virus vacunales. El presente estudio evaluó la interferencia en la protección de la vacuna contra la enfermedad de Newcastle y contra Metapneumovirus en un programa de vacunación simultánea al primer día de edad en pollos de carne.

\section{Materiales y Métodos}

\section{Lugar de Estudio y Animales}

El estudio se realizó en las instalaciones de la unidad de experimentación del Laboratorio de Patología Aviar de la Facultad de Medicina Veterinaria de la Universidad Nacional Mayor de San Marcos (UNMSM), Lima, durante los meses de abril a mayo de 2007. Se utilizaron 400 pollos de carne, mitad machos y mitad hembras, de la línea CobbVantress 500. Las aves fueron criadas en ambientes controlados, donde recibieron el mismo tipo de alimento y agua ad libitum. Las aves se distribuyeron en tres grupos de tratamiento y un grupo control (Cuadro 1).

\section{Reacción Post-vacunal}

Desde el primer día de edad hasta el día 21 se registraron los signos clínicos de estornudos, ronquera, y boqueo. La intensidad de signos clínicos fue considerada como Grado 0: aves normales; Grado 1: Reacción ligera (tos); Grado 2: Reacción moderada (estornudo); Grado 3: Reacción severa (ronquera). El porcentaje de aves con signos clínicos se clasificó como Grado 0: 0\%; Grado 1: hasta $25 \%$; Grado 2: entre 25 a $50 \%$; Grado 3: entre 50 a $75 \%$; y Grado 4: entre 75 a $100 \%$.

\section{Evaluación Serológica}

Se determinaron los niveles de anticuerpos contra ENC, BI y aMPV mediante la prueba de ELISA con kits comerciales (IDEXX Laboratories). Para esto, se tomaron muestras de sangre a 10 aves por grupo en los días 1,30 y 45 de edad. Anticuerpos contra BI y aMPV se determinaron únicamente en el día 45 de edad.

\section{Parámetros Productivos}

Se registró el consumo de alimento y el peso corporal a todas las aves de cada grupo a $\operatorname{los} 7,14,21,28,35,42$ y 45 días de edad. Se analizó el Índice de Conversión Alimenticia (ICA) y el Índice de Eficiencia Productiva Europeo - IEP (Yvore, 1985). Además, se registró la mortalidad diaria y semanal, determinándose la causa de muerte.

\section{Análisis Estadístico}

El peso corporal, consumo de alimento $y$ títulos de anticuerpos se analizaron mediante el análisis de varianza con arreglo factorial. La tasa de mortalidad se analizó con la prueba de Chi cuadrado.

\section{Resultados}

Se presentaron dos tipos de curvas de reacción post vacunal. Los grupos I y II mostraron dos picos de reacción en los días 7 y 12 , y el grupo III un solo pico en el día 10 (Fig. 1), mientras que el grupo sin vacunar no mostró signos respiratorios. Asimismo, el Grupo II presentó signos respiratorios más severos en el segundo pico de reacción vacunal, llegando a afectarse hasta el 95\% de los pollos.

La mortalidad fue de 16.6, 20.0, 33.3 y $84.4 \%$ para los grupos I, II, III y IV, respectivamente. No se encontró diferencias estadísticas significativas en mortalidad por ENC después del reto entre los grupos vacunados, pero sí entre los grupos vacunados y el grupo control $(\mathrm{p}<0.05)$ (Cuadro 2). 
Cuadro 1. Esquema de vacunaciones en pollos de carne ${ }^{1}$

\begin{tabular}{lll}
\hline & $1^{\text {er }}$ día de edad & Día 9 de edad \\
\hline \multirow{3}{*}{ Grupo I } & BI (cepa H120) & ENC (cepa VG/GA) \\
& $\begin{array}{l}\text { ENC (cepa VG/GA) } \\
\text { aMPV (cepa 11/94 origen pollo) }\end{array}$ & \\
Grupo II & $\begin{array}{l}\text { BI (cepa Ma5) } \\
\text { ENC (cepa C2 de tropismo respiratorio) } \\
\text { aMPV (cepa 11/94 origen pollo) }\end{array}$ & ENC (Clone 30) \\
Grupo III & $\begin{array}{l}\text { contra IB (cepa H120) } \\
\text { ENC (cepa VG/GA) }\end{array}$ & ENC (cepa VG/GA) \\
Grupo IV & - & - - - \\
\hline 1 & Al día 30 de edad, todos los grupos fueron desafiados con una cepa velogénica viscerotrópica del \\
virus de Newcastle (vvENC) aislada en el Laboratorio de Patología Aviar - UNMSM
\end{tabular}

Cuadro 2. Porcentaje de mortalidad y de síntomas nerviosos a los 45 días de edad en aves vacunadas $^{1}$ contra Bronquitis Infecciosa (BI), Enfermedad de Newcastle (ENC) y Metapneumovirus (aMPV) al primer día de edad y desafiadas con cepa velogénica viscerotrópica del virus de Newcastle a los 30 días de edad

\begin{tabular}{lcccc}
\hline Parámetro & Grupo I & Grupo II & Grupo III & $\begin{array}{c}\text { Grupo IV } \\
\text { (Control) }\end{array}$ \\
\hline Muertas/total desafiadas & $10 / 85$ & $13 / 85$ & $18 / 78$ & $76 / 86$ \\
Mortalidad (\%) & $12^{\mathrm{a}}$ & $16^{\mathrm{a}}$ & $23^{\mathrm{a}}$ & $88.4^{\mathrm{b}}$ \\
Tics nerviosos & 2.4 & 3.5 & 1.3 & 2.3 \\
Parálisis & 0 & 0 & 0 & 9.3 \\
\hline
\end{tabular}

${ }^{a, b}$ Superíndices diferentes dentro de filas indican diferencia estadística $(p<0.05)$

1 Grupo I: vacunado contra BI (cepa H120), ENC (cepa VG/GA) y aMPV (cepa 11/94 origen pollo); Grupo II: contra BI (cepa Ma5), ENC (cepa C2 de tropismo respiratorio) y aMPV (cepa 11/94 origen pollo); Grupo III: contra IB (cepa H120) y ENC (cepa VG/GA). Todos fueron revacunados contra Newcastle al $9^{\circ}$ día de edad.

El promedio de títulos de anticuerpos maternales para ENC al primer día de edad fue de 5731, nulos en el 30, previo al desafío, y en el día 45 fueron de 5321, 6629, 6435 y 69012 para los grupos I, II, III y IV, respectivamente, sin encontrar diferencias estadísticas significativas entre grupos (Cuadro 3).

La respuesta serológica para $\mathrm{BI}$ al día 45 mostró niveles muy bajos de anticuerpos en todos los grupos, no habiendo diferencia significativa entre grupos vacunados pero sí entre los vacunados y el control (Cuadro 3).
Asimismo, los títulos para aMPV muestran dos grupos serológicos, uno constituido por los Grupos I, II y IV positivos y otro por el Grupo III negativo a la prueba de ELISA (Cuadro 3).

El peso corporal fue similar en todos los grupos hasta el día 28. A los 5 días posteriores al reto, las aves del grupo control mostraron una disminución considerable de peso en comparación con los grupos vacunados; sin embargo, al final del experimento (45 días de edad), las aves sobrevivientes 
Cuadro 3. Títulos de anticuerpos contra el virus de la ENC, BI y mPMV a los 45 días de edad en pollos de carne vacunados al $1^{\text {er }}$ día de edad y revacunados contra Newcastle a los 9 días de edad

\begin{tabular}{lccccc}
\hline & & Grupo I & Grupo II & Grupo III & Grupo IV \\
\hline \multirow{2}{*}{ Enfermedad de } & PAT $^{1}$ & $5616^{\mathrm{a}}$ & $6738^{\mathrm{a}}$ & $6601^{\mathrm{a}}$ & $7258^{\mathrm{a}}$ \\
Newcastle & PGT $^{2}$ & $5321^{\mathrm{a}}$ & $6629^{\mathrm{a}}$ & $6435^{\mathrm{a}}$ & $6902^{\mathrm{a}}$ \\
& $\% \mathrm{CV}$ & 30.1 & 17.0 & 19.9 & 31.0 \\
\multirow{3}{*}{ Bronquitis Infecciosa } & PAT & $128^{\mathrm{a}}$ & $173^{\mathrm{a}}$ & $150^{\mathrm{a}}$ & $73^{\mathrm{b}}$ \\
& PGT & $24^{\mathrm{a}}$ & $112^{\mathrm{a}}$ & $106^{\mathrm{a}}$ & $4^{\mathrm{b}}$ \\
& $\% \mathrm{CV}$ & 175.3 & 71.1 & 80.4 & 270.8 \\
Metapneumovirus & PAT & $4379^{\mathrm{a}}$ & $5507^{\mathrm{a}}$ & $97^{\mathrm{b}}$ & $5691^{\mathrm{a}}$ \\
& PGT & $4253^{\mathrm{a}}$ & $4512^{\mathrm{a}}$ & $17^{\mathrm{b}}$ & $4551^{\mathrm{a}}$ \\
& $\% \mathrm{CV}$ & 24.8 & 64.2 & 125.9 & 74.2 \\
\hline
\end{tabular}

${ }^{a, b}$ Superíndices diferentes dentro de filas indican diferencia estadística $(p<0.05)$

${ }^{1}$ Promedio aritmético total

2 PGT: Promedio Geométrico Total

Cuadro 4. Peso corporal semanal en pollos de carne (gramos) vacunados al $1^{\text {er }}$ día de edad contra ENC, BI y mPMV y revacunados contra Newcastle a los 9 días de edad

\begin{tabular}{lcccc}
\hline Edad & Grupo I & Grupo II & Grupo III & Grupo IV \\
\hline Inicio & $45.9^{\mathrm{a}}$ & $47.3^{\mathrm{a}}$ & $46.1^{\mathrm{a}}$ & $46.7^{\mathrm{a}}$ \\
$1^{\mathrm{o}}$ semana & $162.6^{\mathrm{a}}$ & $183.6^{\mathrm{b}}$ & $166.3^{\mathrm{a}}$ & $189.6^{\mathrm{b}}$ \\
$2^{\mathrm{o}}$ semana & $402.5^{\mathrm{ab}}$ & $427.5^{\mathrm{bc}}$ & $397.0^{\mathrm{a}}$ & $450.1^{\mathrm{c}}$ \\
$3^{\mathrm{o}}$ semana & $791.6^{\mathrm{a}}$ & $851.0^{\mathrm{bc}}$ & $824.5^{\mathrm{ab}}$ & $890.9^{\mathrm{c}}$ \\
$4^{\mathrm{o}}$ semana & $1310.3^{\mathrm{a}}$ & $1356.4^{\mathrm{a}}$ & $1335.8^{\mathrm{a}}$ & $1365.8^{\mathrm{a}}$ \\
$5^{\mathrm{o}}$ semana & $1761.4^{\mathrm{a}}$ & $1917.3^{\mathrm{b}}$ & $1812.1^{\mathrm{ab}}$ & $1672.0^{\mathrm{a}}$ \\
$6^{\circ}$ semana & $2339.0^{\mathrm{a}}$ & $2447.2^{\mathrm{a}}$ & $2446.9^{\mathrm{a}}$ & $2335.6^{\mathrm{a}}$ \\
45 días & $2565.6^{\mathrm{a}}$ & $2621.0^{\mathrm{a}}$ & $2701.5^{\mathrm{a}}$ & $2785.7^{\mathrm{a}}$ \\
\hline
\end{tabular}

${ }^{a, b}$ Superíndices diferentes dentro de filas indican diferencia estadística $(p<0.05)$

${ }^{1}$ Grupo I: vacunado contra BI (cepa H120), ENC (cepa VG/GA) y aMPV (cepa 11/94 origen pollo); Grupo II: contra BI (cepa Ma5), ENC (cepa C2 de tropismo respiratorio) y aMPV (cepa 11/94 origen pollo); Grupo III: contra IB (cepa H120) y ENC (cepa VG/GA). Todos fueron revacunados contra Newcastle al $9^{\circ}$ día de edad.

de este grupo presentaron un relativo mayor peso corporal, aunque sin diferencias estadísticas entre grupos (Cuadro 4). Las aves del grupo I mostraron el mejor ICA y el mejor IEP (Cuadro 5).

\section{Discusión}

Todos los grupos de aves vacunadas presentaron reacciones respiratorias postvacunales de intensidad variable, lo cual tuvo 
Cuadro 5. Comportamiento productivo a los 45 días de edad en pollos de carne vacunados al $1^{\text {er }}$ día de edad contra ENC, BI y mPMV y revacunados contra Newcastle a los 9 días de edad

\begin{tabular}{ccccc}
\hline Grupo $^{1}$ & Peso Final & I.C.A. $^{2}$ & Viabilidad & I.E.P.E. $^{3}$ \\
\hline I & 2.57 & 2.34 & 83.3 & 203.0 \\
II & 2.62 & 2.44 & 80.0 & 191.0 \\
III & 2.70 & 2.54 & 71.1 & 168.0 \\
IV & 2.79 & 10.58 & 15.6 & 9.1 \\
\hline
\end{tabular}

${ }^{1}$ Grupo I: vacunado contra BI (cepa H120), ENC (cepa VG/GA) y aMPV (cepa 11/94 origen pollo); Grupo II: contra BI (cepa Ma5), ENC (cepa C2 de tropismo respiratorio) y aMPV (cepa 11/94 origen pollo); Grupo III: contra IB (cepa H120) y ENC (cepa VG/GA). Todos fueron revacunados contra Newcastle al $9^{\circ}$ día de edad.

2 Índice de Conversión Alimenticia

3 Índice de Eficiencia Productivo Europeo

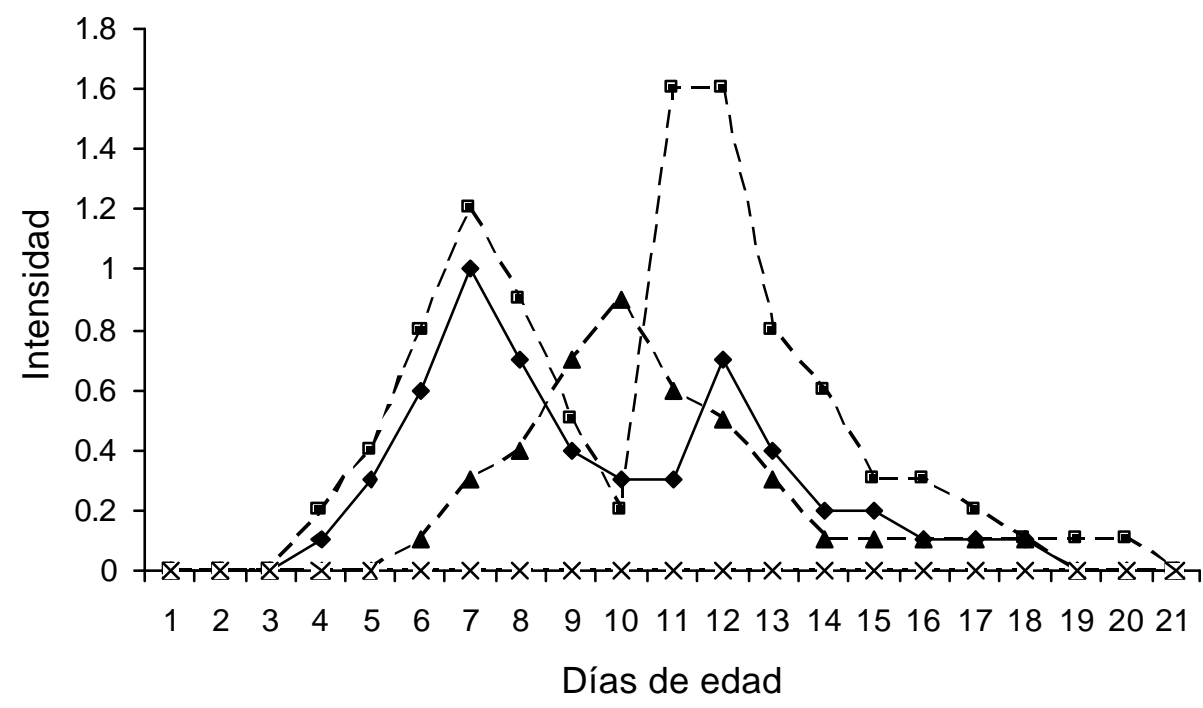

Figura 1. Grados de reacciones respiratorias audibles (estornudos y ronquera) en los primeros 21 días de edad en pollos de carne vacunados al primer día de edad contra Bronquitis Infecciosa, Enfermedad de Newcastle y Metapneumovirus. Grupo I: contra BI (cepa H120), ENC (cepa VG/GA) y aMPV (cepa 11/94 origen pollo); Grupo II: contra BI (cepa Ma5), ENC (cepa C2 de tropismo respiratorio) y aMPV (cepa 11/94 origen pollo); Grupo III: contra IB (cepa H120) y ENC (cepa VG/GA). Todos fueron revacunado al $9^{\circ}$ día contra ENC.

relación con el programa vacunal y el tipo de cepa usada en la vacuna contra ENC. Los grupos I y II presentaron dos picos bien diferenciados de reacciones respiratorias audibles a los 7 y 12 días de edad. El primer pico fue en reacción a la vacunación con
aMPV y el segundo, mucho más pronunciado en el grupo II, fue debido a la revacunación contra ENC (cepa respiratoria). Esto demuestra que cuando se usa en la vacunación simultánea una cepa vacunal de ENC de tropismo respiratorio se incrementa la severidad de las 
reacciones respiratorias post vacunales. Los grupos I y III, vacunados con cepa entérica de ENC, tuvieron reacciones menos severas, consideradas como normales y consistentes con el uso de vacunas menos invasivas. La ausencia de un segundo pico en el grupo II se debió a que no se vacunó contra aMPV.

La respuesta serológica de los grupos fue de acuerdo a lo esperado. El primer día, títulos altos para Newcastle debido a la inmunidad pasiva proveniente de la madre y el día 30 con títulos mínimos o nulos de anticuerpos, lo cual está de acuerdo con el silencio inmunológico demostrado en las aves a esta edad (Davison et al., 2008). Al día 45, los cuatro grupos experimentales mostraron elevados títulos de anticuerpos contra el virus de la enfermedad de Newcastle como consecuencia del desafío con el virus velogénico.

La respuesta serológica humoral contra el virus de la BI a los 45 días de edad fue mínima, debido a que las aves recibieron solo una vacuna viva al primer de edad. Aun cuando los grupos vacunados fueron considerados como seronegativos, el grupo control, no vacunado, mostró el menor título y el mayor coeficiente de variación $(\mathrm{p}<0.05)$.

Los resultados de la prueba de ELISA para Metapneumovirus aviar a los 45 días de edad en los grupos I, II y III estuvieron de acuerdo con el programa de vacunación usado. Los grupos I y II vacunados contra este agente presentaron títulos altos, y el grupo III no vacunado contra aMPV fue negativo; sin embargo, los resultados del grupo IV control no vacunado resultaron controversiales, ya que se obtuvo seroconversión con alto título contra el aMPV y elevado coeficiente de variación, demostrando contaminación horizontal del virus vacunal al final del experimento a partir de los grupos vacunados. $\mathrm{La}$ transmisibilidad horizontal del virus ha sido demostrada en otros estudios (Jones, 2002).

Las aves de todos los grupos mostraron signos clínicos a partir del primer día del reto con la cepa vvENC, inicialmente de tipo res piratorio, luego digestivo (diarrea), y por último nervioso, ocurriendo casos de mortalidad a partir del $4^{\circ}$ día post desafío. Estudios previos reportaron similares resultados (Alva, 2001).

No se encontró diferencia estadística en el peso corporal entre grupos experimentales previo al desafío. Posteriormente, el grupo control, perdió peso pero logró desarrollar un crecimiento compensatorio, el cual ha sido demostrado en aves sobrevivientes a enfermedades con alta conversión alimenticia (Jones, 2002).

La mejor conversión alimenticia fue observada en los grupos I y II vacunados contra aMPV, siendo el grupo I ligeramente mejor que el grupo II debido al uso de una vacuna de Newcastle con cepa entérica. El grupo III mostró una conversión ligeramente más deficiente que el grupo II, debido, probablemente, a un problema de mortalidad inicial por onfalitis que afectó a las aves de este grupo en la primera semana. El grupo control, no vacunado, mostró la peor conversión alimenticia debido a los efectos de la enfermedad de Newcastle, especialmente por la alta mortalidad ocasionada en este grupo $(88 \%)$.

El Grupo I tuvo un mejor desempeño productivo y menor mortalidad, así como una reacción post-vacunal más leve que el Grupo II, debido al uso de la cepa vacunal entérica de Newcastle, la cual habría dejado más células diana traqueales libres para los otros virus vacunales (Ganapathy et al., 2005, 2006).

El desempeño global de los grupos estimado de acuerdo al IEP estuvo en relación con la cantidad y severidad de los signos clínicos ocasionados por el desafío con el virus de la ENC. Todos los grupos vacunados mostraron protección sin diferencia significativa frente al desafío con virus patógeno de ENC al día 45; sin embargo, el grupo I, vacunado dos veces con una cepa de Newcastle enterotrópica y aMPV con BI al primer día, mostró una menor reacción post-vacunal, una menor mortalidad y, numéricamente, mejor IEP. 


\section{Conclusiones}

? La vacunación contra Metapneumovirus Aviar (aMPV) en forma simultánea contra la enfermedad de Newcastle (ENC) y Bronquitis Infecciosa (BI) al primer día de edad en pollos de carne no ocasionó interferencia en la protección contra la Enfermedad de Newcastle en ninguno de los grupos vacunados.

? Se obtuvo menor reacción post vacunal con la vacunación con cepa entérica del virus de la Enfermedad de Newcastle en comparación con el uso de la cepa de tropismo respiratorio.

\section{Literatura Citada}

1. Alva B. 2001. Newcastle velogénico, siempre un tema de actualidad. En: Memorias XXIV Reunión Científica Anual de la Asociación Peruana de Producción Animal. Lima, Perú: APPA.

2. Bennett RS, Nezworski J, Velayudhan BT, Nagaraja KV, Zeman DH, Dyer $N$, Graham T, Lauer DC, Nenga MK, Halvorson DA. 2001. Evidence of avian pneumovirus spread beyond Minnesota among wild and domestic birds in central North America. Avian Dis 48: 902908.

3. Cook JK. 2001. Infectious bronchitis virus vaccine interferes with the replication of avian Metapneumovirus vaccine in domestic fowl. Avian Pathol 30: 233-242.

4. Davison F, Kaspers B, Schat KA. 2008. Avian immunology. London: Academic Press. 481 p.

5. Ganapathy K, Cargill P, Montiel E, Jones $R C$. 2006. Interaction between live avian Metapneumovirus and Newcastle disease virus vaccines in specific pathogen free chickens. Avian Pathol 34: 297-302.
6. Ganapathy K, Todd V, Cargill $P$, Montiel E, Jones RC. 2006 Interaction between a live avian Metapneumovirus vaccine and two different Newcastle disease virus vaccines in broiler chickens with maternal antibodies to Newcastle disease virus. Avian Pathol 35: 429-434.

7. Gross WB. 1994. Disease due to Escherichia coli in poultry. In: Escherichia coli in domestic animals and humans. Gyles CL (ed). Wallingford, United Kingdom: CAB Internacional. $p$ 237-259.

8. Icochea E. 2002. Infección por Pneumovirus aviar. En: Memorias I Seminario Asociación de Médicos Veterinarios Especialistas en Aves. Lima, Perú: AMEVEA.

9. Yvore P. 1985. The use of shuttle programs for the control of coccidiosis in broilers. Proc. Gerogia Coccidiosis Conf. Georgia, USA.

10. Jones RC. 2002. Infección por Pneumovirus aviar en pollos de engorde y ponedoras. X Congreso de Patología Aviar. Athens, Georgia, EEUU.

11. Jones RC. 2004. Avian Metapneumovirus infections in chickens and turkeys. En: Memorias II Seminario Internacional Asociación de Médicos Veterinarios Especialistas en Aves. Lima, Perú: AMEVEA.

12. Morley AJ, Thompson DK. 1984. Swollen head syndrome in broiler chickens. Avian Dis 28: 238-243.

13. Shin HJ, Jirjis FF, Kumar MC, Njenga MK, Shaw DP, Noll SL, Nagaraja KV, Halvorson DA. 2002. Neonatal avian pneumovirus infection in commercial turkeys. Avian Dis 46: 239244.

14. Villegas P. 2009. Rol de los virus de Bronquitis Infecciosa y Metapneumovirus Aviar en los problemas respiratorios de pollos de carne. Prevención y control. En: V Seminario Internacional Asociación de Médicos Veterinarios Especialistas en Aves. Lima: AMEVEA. 